\title{
New Targeted Therapies Against Breast Cancer
}

\author{
Nicolin Vanessa*, Bareggi Renato and Narducci Paola \\ Section of Molecular Biology, Clinical Department of Biomedicine, University of Trieste Via Manzoni, 1634138 Trieste, Italy
}

\begin{abstract}
Breast cancer is one of the most frequently diagnosed cancers among women, with an estimated 184,450 new cases of invasive disease and 40,930 deaths in 2008. There is strong evidence that estrogen plays a role in its development and progression. Breast cancer remains a significant public health problem despite advances in the understanding of the molecular and cellular events that underlie the disease. Crucial pathways regulating the cell cycle, proliferation and survival of breast cancer cells have been investigated and aberrant components of these pathways have been exploited as new drug targets.
\end{abstract}

Keywords: Breast cancer; Bone osteolytic metastasis; RANKL; New drugs

\section{Membrane-associated Estrogen Receptor Signaling Pathways in Human Breast Cancer}

The diagnosis of breast cancer falls into two broad categories, either estrogen receptor (ER)-positive or ER-negative, based on the level of ER in the cancer cells [1]. ER is expressed in about $60 \%$ of all breast cancers. ER-positive breast cancer generally has a better prognosis, and is often responsive to antiestrogen therapy [2]. In contrast, ER-independent breast cancers are more aggressive and unresponsive to antiestrogens [3]. Although estrogen does not appear to directly cause the DNA mutations that trigger the development of human cancer, estrogen does stimulate cell proliferation [4]. Therefore, if one or more breast cells already possesses a DNA mutation that increases the risk of developing cancer, these cells will proliferate (along with normal breast cells) in response to estrogen stimulation (Figure 1). The result will be an increase in the total number of mutant cells, any of which might thereafter acquire the additional mutations that lead to uncontrolled proliferation and the onset of cancer. In other words, estrogen-induced cell production leads to an increase in the total number of mutant cells that exist [5]. These cells are at increased risk of becoming cancerous, so the chances that cancer may actually develop are increased. Even in women who do not have any mutant breast cells, estrogen-induced proliferation of normal breast cells may still increase the risk of developing cancer. Proliferation of normal cells from exposure to estrogen creates a vulnerability to spontaneous mutations, some of which might represent a first step on the pathway to cancer (Figure 2).

\section{Targeted Therapy of Metastatic Breast Cancer}

Breast cancer is a heterogeneous disease characterised by a dysregulation of multiple pathways related to cell differentiation, cell cycle control, apoptosis, angiogenesis and development of metastasis. Acting against these pathways provides therapeutic targets for new targeted biologic therapies, which, in the future, might constitute a key for fighting cancer [6,7]. The development of molecular technology in recent years has allowed a further comprehension of these mutations and dysregulated pathways leading to oncogenesis. New targeted biologic therapies will block essential functions of cancer cells and tumour stroma [8]. A growing number of therapy options, alone or in combination with background treatments (chemotherapy, hormone therapy, radiotherapy), will allow oncologists a better adaptation of treatment to patients and disease characteristics. Examples of approved targeted agents in breast cancer include agents targeting the human epidermal growth factor receptor 2 (HER2), such as trastuzumab and lapatinib $[8,9]$. Lapatinib is a unique, orally bioavailable, small-molecule dual tyrosine kinase inhibitor that targets tumor cells overexpressing both human epidermal growth factor receptor (EGFR; ErbB1) and ErbB2 tyrosine kinases. Lapatinib inhibition of ErbB1 and ErbB2 kinase activity prevents the activation of downstream cellular signals that promote tumor cell survival and proliferation. Using a rational drug design approach, more than 3200 quinazoline and quinazoline-like compounds with potential tyrosine kinase activity were screened and assayed. Lapatinib was eventually selected from these compounds as it was a selective and potent inhibitor of ErbB1 and ErbB2 that had predictable oral bioavailability and acceptable in vivo toxicity in the targeted patient population. Initial studies in humans with lapatinib were started in 2001; in 2007 lapatinib was approved in the USA for use in combination with capecitabine for the treatment

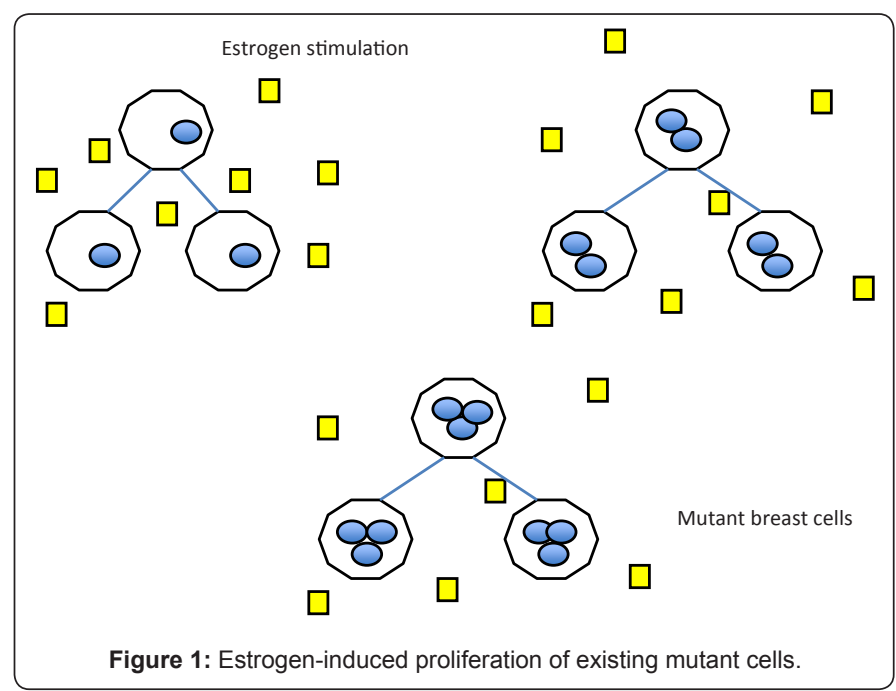

Corresponding author: Nicolin Vanessa, PhD, Assistant Professor, Section of Molecular Biology, Clinical Department of Biomedicine, University of Trieste, Via Manzoni, 1634138 Trieste, E-mail: nicolin@units.it

Received November 09, 2010; Accepted December 04, 2010; Published December 06, 2010

Citation: Nicolin V, Bareggi R, Paola N (2010) New Targeted Therapies Against Breast Cancer. J Carcinogene Mutagene 1:110. doi:10.4172/2157-2518.1000110

Copyright: @ 2010 Nicolin V, et al. This is an open-access article distributed unde the terms of the Creative Commons Attribution License, which permits unrestricted use, distribution, and reproduction in any medium, provided the original author and source are credited. 


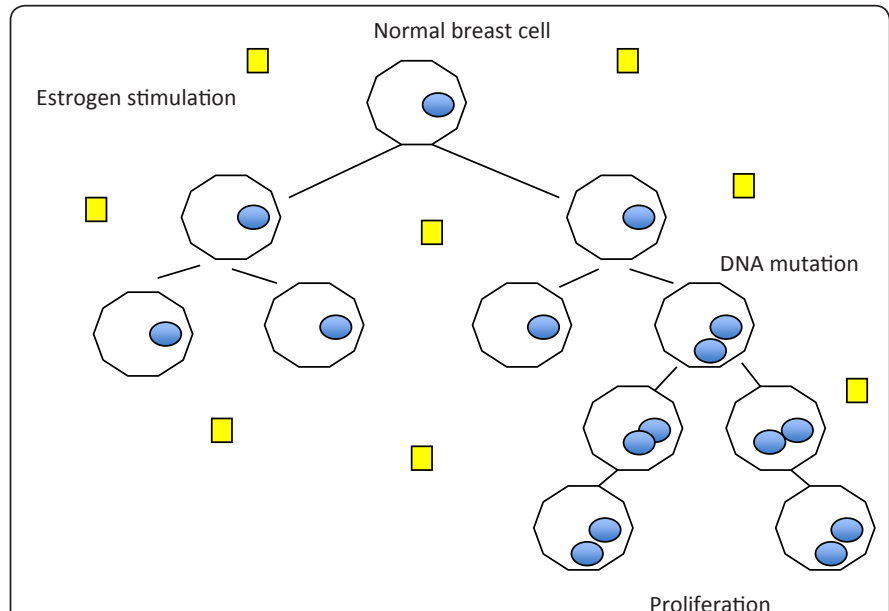

Figure 2: Estrogen-induced proliferation and spontaneous new mutations.

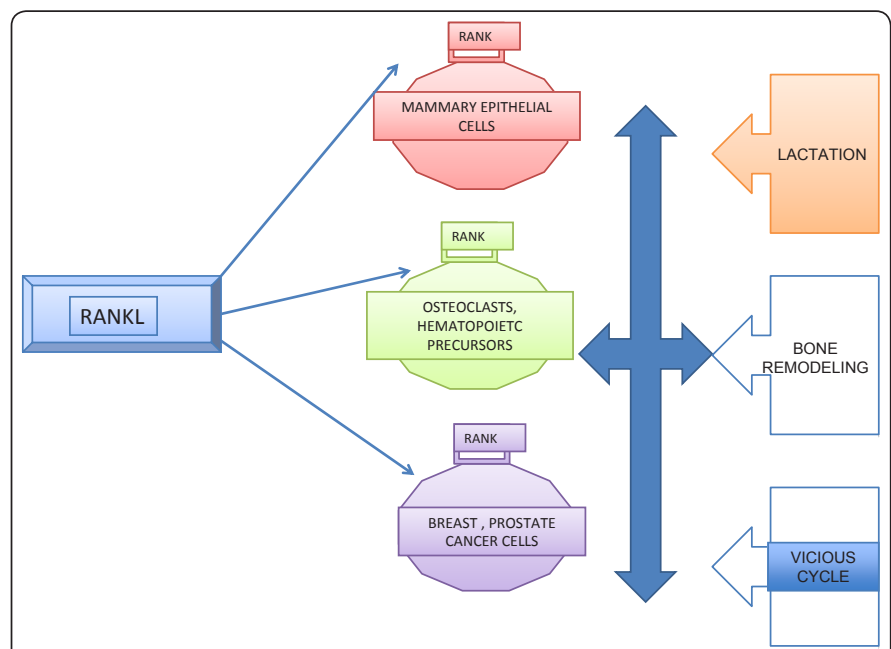

Figure 3: Role of RANKL in lactation, bone remodeling and bone osteolysis induced by breast cancer (Narducci, 2009).

of ErbB2 + advanced or metastatic breast cancer in patients who had received previous treatment including an anthracycline, a taxane and trastuzumab.In addition, there are other therapy classes under evaluation, including novel anti-EGFR or anti-HER2 therapies; agents fighting other tyrosine kinases, including the Src and the insulin-like growth factor receptor; agents interfering critically relevant pathways, such as PI3K/AKT/mTOR inhibitors; and agents promoting apoptosis, such as PARP inhibitors (for particular breast cancer subtypes, such as basal-like, or breast cancer with BRCA mutations) and others.

\section{Breast Cancer and Bone Osteolysis}

Bone is a highly hospitable environment for colonization and growth of metastatic tumors, and some of the most common human malignancies, notably breast cancer and prostate cancer, have a strong propensity to produce skeletal metastases [10]. Tumor cells, in turn, can produce a spectrum of skeletal manifestations which includes diffuse osteopenia, focal osteolysis, focal osteogenesis, and osteomalacia [11]. Since the late nineteenth century, it has been thought that the microenvironment of the local host tissue actively participates in the propensity of certain cancers to metastasize to specific organs, and that bone provides an especially fertile 'soil' $[12,13]$. In the case of breast cancers, the local chemokine milieu is now emerging as an explanation for why these tumors preferentially metastasize to certain organs. In order for tumor cells to grow and invade mineralized bone, osteolysis must occur. Osteoclasts appear uniquely adapted to produce the microenvironment and the biochemical milieu that are needed to resorb bone. Although previous reports have indicated that some tumor cells appear capable of assuming an osteoclast phenotype and directly resorbing bone the bulk of the evidence suggests that most tumor cells act indirectly by co-opting the physiologic mechanisms that normally favor bone resorption. Thus, they release agents such as hormones, eicosanoids, growth factors, and cytokines into the bone microenvironment, which act on osteoblastic stromal cells to enhance the production of osteoclast activating factors. Most notable of these is the cell membrane-associated protein termed receptor activator of NF- $\mathrm{KB}$ ligand (RANKL), which is a member of the TNF family of cytokines. RANKL can then bind to its cognate receptor (RANK) on osteoclast precursors and, in the presence of M-CSF, enhance the differentiation and fusion of these cells to produce functioning multinucleated osteoclasts (Figure 3). Accumulating evidence has shown that RANKL is not only important for normal bone development, but is implicate in a spectrum of skeletal diseases characterized by excessive osteoclastic activity, including osteoporosis, rheumatoid arthritis, and bone metastases. The typical tumor-induced bone lesion is focal osteolysis, although some tumors such as breast cancer metastases exhibit osteogenic bone lesions. The RANKL/RANK pathway may direct breast cancer cells to preferentially migrate into bone, the crucial requirement and initial step for skeletal metastasis. Epithelial cells from normal mammary glands express RANK, and RANKL-RANK signalling is required for the development of lactating mammary glands during pregnancy. Both RANKL- and RANK-deficient mice lack lactating mammary glands and cannot feed their off-spring. Based on high constitutive RANK expression in breast cancer specimens and cell lines, recent data now indicate that the RANK expression status of cancer cells determines whether tumors predominantly migrate into bone, where the corresponding ligand RANKL is abundantly expressed. The correlation of high RANK expression with osteotropism in murine models was demonstrated across diverse tumor cell types, including breast cancer and melanoma [13]. The osteolysis induced by these tumors affects the quality of life of the patients by causing pathological fractures and severe bone pain and often exacerbates the prognosis of the patients. Concomitantly, production of a soluble decoy receptor for RANKL, termed osteoprotegerin (OPG), may be downregulated, thus eliminating one means by which the ensuing osteolysis could be repressed. The imbalance in the RANKL: OPG ratio that results in a RANKL upregulation and expression leads to increased osteoclastogenesis and consequently bone loss [14].

\section{New Therapies for Breast Cancer Induced Osteolysis}

Bone metastases significantly limit patients' quality of life and life expectancy. Cancer patients with bone metastases have a high risk of skeletal morbidity. They have reduced mobility, pain, and bone weakness that predisposes them to pathologic fractures, spinal epidural compression, and bone marrow failure. Common primary tumors that can cause bone metastases include breast, prostate, lung, colon, stomach, bladder, uterine, rectal, thyroid, and kidney cancers. Hematologic malignancies such as multiple myeloma and non-Hodgkin's lymphoma frequently involve bones and can increase the risk of pathologic fractures and spinal cord compression syndromes. Breast cancer can metastasize to bone in about $75 \%$ of cases of advanced disease and usually causes osteolytic destruction. Bony metastases in patients with breast cancer lead to elevated bone 
turnover markers such as the urinary $\mathrm{N}$-telopeptide corrected for urinary creatinine (uNTx/Cr); patients with elevated levels of $u N T x / C r$ are at increased risk for skeletal complications, disease progression, and death. Denosumab is a fully human monoclonal antibody to the receptor activator of nuclear factor $\kappa B$ ligand (RANKL) [15]. RANKL is an important mediator of osteoclast differentiation, function, and survival and plays a central role in bone destruction related to metastatic cancer. Tumor cell factors in bone stimulate expression of RANKL, which binds to RANK on precursor and mature osteoclasts. Osteoclast-mediated bone resorption spurs the production of growth factors that further stimulate tumor growth, resulting in a cycle of bone destruction and tumor cell proliferation. RANKL also has been found to promote the migration of RANK expressing tumor cells to bone. By inhibiting RANKL, denosumab inhibits osteoclast function and bone resorption $[16,17,18]$.

\section{Cancer Chemopreventive Potential of Natural Products}

Epidemiologic studies have suggested that antioxidant supplements might reduce the risk of breast cancer recurrence or breast cancer-related mortality [19]. Many naturally occurring agents have shown cancer chemopreventive potential in a variety of bioassay systems and animal models with relevance to human disease; e.g., genistein, one of the major soy isoflavones, has antioxidant properties, is a potent inhibitor of angiogenesis and metastasis and is a promising reagent for cancer chemoprevention and/or treatment [21]. Danshen (Salvia miltiorrhiza Bunge) is a widely used Chinese herbal medicine; its extracts contain diterpene quinone and phenolic acid derivatives, including tanshinone (I, IIA and IIB, $\mathrm{VI}$ ), cryptotanshinone, isocryptotanshinone, miltirone, tanshinol (I and II) and salviol $[21,22,23]$. These compounds have antioxidant properties and protect against lipid peroxidation in vitro and in vivo, making them potential antidotes for free radical-based disorders $[24,25,26]$. Tanshinone IIA is a derivative of phenanthrene-quinone, isolated from Danshen; it has antioxidant properties, inhibiting the association of lipid peroxidation products with DNA by breaking the chain reactions of peroxidation by scavenging lipid free radicals. The planar phenanthrene ring of the tanshinones may be essential for interaction with DNA molecules, whereas the furano-o-quinone moiety could be responsible for the production of reactive free radicals in the close vicinity of bases that causes DNA damage, giving tanshinone cytotoxic activity against multiple human cancer cell lines. Studies in vitro have shown that tanshinone IIA inhibited the proliferation of mouse P388 lymphocytic leukemia cells [26] and human hepatoma cells [23,27] induced apoptosis of human hepatocellular carcinoma cells, human promyelocytic leukemic cells and human erythroleukemic cells (K562); and induced differentiation of human leukemia cells [28].

\section{References}

1. Clark GM, Osborne CK, McGuire WL (1984) Correlations between estrogen receptor, progesterone receptor, and patient characteristics in human breast cancer. J Clin Oncol 2: 1102-1109.

2. Russo IH, Russo J (1998) Role of hormones in mammary cancer initiation and progression. J Mammary Gland Biol Neoplasia 3: 49-61.

3. Shoker BS, Jarvis C, Clarke RB, Anderson E, Hewlett J, et al. (1999) Estrogen receptor-positive proliferating cells in the normal and precancerous breast. Am J Pathol 155: 1811-1815

4. Goldhirsch A, Wood WC, Gelber RD, Coates AS, Thürlimann B, et al. (2007) Progress and promise: highlights of the international expert consensus on the primary therapy of early breast cancer 2007. Ann Oncol 18: 1133-1144.

5. Nemere I, Pietras RJ, Blackmore PF (2003) Membrane receptors for steroid hormones: signal transduction and physiological significance. J Cell Biochem 88: $438-455$.

6. Alvarez RH, Valero V, Hortobagyi GN (2010) Emerging targeted therapies for breast cancer. J Clin Oncol 28: 3366-3379.

7. Maughan KL, Lutterbie MA, Ham PS (2010) Treatment of breast cancer. Am Fam Physician 81: 1339-1346.

8. Viale G, Ghioni M, Matstropasqua MG (2010) Traditional molecular markers and response to adjuvant endocrine or trastuzumab-based therapies. Curr Opin Oncol 22: 541-546.

9. Curran MP (2010) Lapatinib: in postmenopausal women with hormone receptorpositive, HER2-positive metastatic breast cancer. Drugs 70: 1411-1422.

10. Cicek M, Oursler MJ (2006) Breast cancer bone metastasis and current smal therapeutics. Cancer Metatastasis Rev 25: 635-644.

11. Yoneda T, Hiraga T (2005) Crosstalk between cancer cells and bone microenvironment in bone metastasis. Biochem Biophys Res Commun 328: 679-687.

12. Thomas RJ, Guise TA, Yin JJ, Elliott J, Horwood NJ, et al. (1999) Breast cancer cells interact with osteoblasts to support osteoclast formation. Endocrinology 140: $4451-4458$

13. Nicolin V, Bortul R, Bareggi R, Baldini G, Martinelli B, et al. (2008) Breast adenocarcinoma MCF-7 cell line induces spontaneous osteoclastogenesis via a RANK-ligand-dependent pathway. Acta Histochem 110: 388-396.

14. Narducci P, Bareggi R, Nicolin V (2009) Receptor activator for nuclear factor kappa $\mathrm{B}$ ligand (RANKL) as an osteoimmune key regulator in bone physiology and pathology. Acta Histochem [Epub ahead of print].

15. Stopeck A (2010) Denosumab findings in metastatic breast cancer. Clin Adv Hematol Oncol 8: 159-160.

16. Bartsch R, De Vries C, Pluschnig U, Dubsky P, Bago-Horvath Z, et al. (2009) Predicting for activity of second-line trastuzumab-based therapy in her2positive advanced breast cancer. BMC Cancer 9: 367.

17. Burkiewicz JS, Scarpace SL, Bruce SP (2009) Denosumab in osteoporosis and oncology. Ann Pharmacother 43: 1445-1455

18. Hamdy NA (2008) Denosumab: RANKL inhibition in the management of bone loss. Drugs Today (Barc) 44: 7-21.

19. Sarkar FH, Li Y (2003) Soy isoflavones and cancer prevention. Cancer Invest 21: 744-757

20. Han H, Zhao Y, Cuthbertson T, Hartman RF, Rose SD (2010) Cell cycle arrest and apoptosis induction by an anticancer chalcone epoxide. Arch Pharm (Weinheim) 343: 429-439.

21. Wu WL, Chang WL, Chen CF (1991) Cytotoxic activities of tanshinones against human carcinoma cell lines. Am J Chin Med 19: 207-216.

22. Niu XL, Ichimori K, Yang X, Hirota Y, Hoshiai K, et al. (2000) Tanshinone II-A inhibits low-density lipoprotein oxidation in vitro. Free Radic Res 33: 305-312.

23. Wang AM, Sha SH, Lesniak W, Schacht J (2003) Tanshinone (Salviae miltiorrhizae extract) preparations attenuate aminoglycoside-induced free radical formation in vitro and ototoxicity in vivo. Antimicrob Agents Chemother 47: 1836-1841.

24. Cao EH, Liu XQ, Wang JJ, Xu NF (1996) Effect of natural antioxidant tanshinone II-A on DNA damage by lipid peroxidation in liver cells. Free Radic Biol Med 20: 801-806.

25. Zhou GY, Zhao BL, Hou JW, Ma GE, Xin WJ (1999) Protective effects of sodium tanshinone IIA sulphonate against Adriamycin-induced lipid peroxidation in mice hearts in vivo and in vitro. Pharmacol Res 40: 487-491.

26. Mosaddik MA (2010) In vitro cytotoxicity of tanshinones isolated from Salvia miltiorrhiza Bunge against P388 lymphocytic leukemia cells. Phytomedicine 10: 682-685.

27. Yuxian X, Feng T, Ren L, Zhengcai L (2009) Tanshinone II-A inhibits invasion and metastasis of human hepatocellular carcinoma cells in vitro and in vivo. Tumori 95: 789-795.

28. Liu XD, Fan RF, Zhang Y, Yang HZ, Fang ZG, et al. (2010) Down-regulation of telomerase activity and activation of caspase- 3 are responsible for tanshinone I-induced apoptosis in monocyte leukemia cells in vitro. Int J Mol Sci 11: 2267 2280. 